\title{
Sexarca, informação e uso de métodos contraceptivos: comparação entre adolescentes
}

\author{
Naiana Dapieve Patias - Universidade Federal de Santa Maria, Santa Maria, Rio Grande do Sul, Brasil \\ Ana Cristina Garcia Dias - Universidade Federal de Santa Maria, Santa Maria, Rio Grande do Sul, Brasil
}

\begin{abstract}
Resumo
Este trabalho teve por objetivo comparar a idade da primeira relação sexual, a informação sobre métodos contraceptivos, além de seu uso com a ocorrência da gestação na adolescência em dois grupos (50 grávidas e 50 não grávidas). Foi utilizado um questionário desenvolvido para o estudo que investigou essas variáveis. Verificou-se que não há diferença significativa nos grupos entre idade da sexarca, uso de contracepção na sexarca e tipo de contraceptivo utilizado. Contudo, os grupos diferem em relação ao uso de métodos contraceptivos nas relações sexuais subsequentes. Os dados revelam a multiplicidade de fatores envolvidos no comportamento contraceptivo demonstrando a necessidade de absorção desses fatores por parte das políticas públicas voltadas para o público adolescentes.

Paluras-chave: Gravidez; Adolescência; Sexarca; Contracepção.
\end{abstract}

First sexual intercourse, information and contraceptive use: a comparison between adolescents

\begin{abstract}
This study aimed to compare the age at first sexual intercourse, information about contraceptive methods, besides its use with the occurrence of adolescent pregnancy in two groups (50 pregnant and 50 nonpregnant). The questionnaire developed for the study investigated these variables. It was found that there is no significant difference between groups in age at first sexual intercourse, use of contraception at first sexual intercourse and type of contraceptive used. However, the groups differ with respect to the use of contraceptives in the subsequent sexual intercourses. The data reveals the multiplicity of factors involved in contraceptive behavior demonstrating the need for absorption of these factors on the part of public politics directed to adolescents. Keywords: Pregnancy, Adolescence, First sexual intercourse, Contraception.
\end{abstract}

\section{Sexarca, información y uso de métodos anticonceptivos: comparación entre adolescentes}

\section{Resumen}

Este estudio tuvo como objetivo comparar la edad de la primera relación sexual, la información sobre los métodos anticonceptivos, además de su uso con la ocurrencia del embarazo en la adolescencia en dos grupos (50 embarazadas y 50 no embarazadas). El cuestionario elaborado para el estudio investigó esas variables. Se encontró que no hay diferencia significativa en los grupos entre edad de la sexarca, el uso de anticonceptivos en la sexarca y el tipo de anticonceptivo utilizado. Sin embargo, los grupos difieren con respecto a la utilización de anticonceptivos en las relaciones sexuales posteriores. Los datos revelan la multiplicidad de factores implicados en el comportamiento anticonceptivo que demuestran la necesidad de absorción de estos factores en las políticas públicas vueltas a los adolescentes.

Palabras clave: Embarazo, Adolescencia, Sexarca, Anticoncepción.

\section{Introdução}

As mudanças da puberdade sinalizam que os indivíduos estão biologicamente capacitados à reprodução (Cano, Ferriani, \& Gomes, 2000). Contudo, isso não quer dizer que estejam psiquicamente preparados para o exercício sexual e para a parentalidade. Nesse contexto, relações sexuais desprotegidas podem gerar tanto doenças sexualmente trasmissíveis (DSTs), como a ocorrência de uma gestação indesejada (Brandão, 2009; Júnior e cols., 2009). Essa vulnerabilidade à gestação e às DSTs decorre de inúmeros fatores, tanto individuais (motivacionais), como contextuais (sociais e culturais), que estão relacionados à sexualidade.

Entre esses fatores podem ser destacados: as características da própria adolescência, como a impulsividade e o pensamento egocêntrico (Steinberg, 2010); a idade da primeira relação sexual (sexarca) (Ximenes Neto, Dias, Rocha, \& Cunhas, 2007), a falta de informação sobre contraceptivos; o uso infrequente ou inadequado de métodos contraceptivos, (Oliveira, Dias, \& Silva, 2005; Santos \& Carvalho, 2006; Villela \& Doretto, 2006); estar ou não frequentando a escola (Cruzeiro e cols., 2010; Leite, Rodrigues, \& Fonseca, 2004); o número de parceiros sexuais (Charles \& Blum, 2008; Kotchick, Shaffer, Forehand, \& Miller, 2001; Luster \& Small, 1994; Taquette \& Vilhena, 2008), além disso, motivações pessoais como a crença de que métodos contraceptivos podem engordar, diminuir o prazer ou até mesmo mostrar que a menina que utiliza o contraceptivo estaria "preparada" para ter relações sexuais aliam-se a crenças e concepções tradicionais de gênero que podem interferir na adoção ou 
não de métodos contraceptivos (Asinelli-Luz \& Junior, 2008; Carvalho \& Schor, 2005; Taquette, Vilhena \& Paula, 2004).

Este estudo objetivou verificar se há diferenças entre dois grupos de adolescentes (gestantes e não gestantes) em algumas dessas variáveis: idade da primeira relação sexual (sexarca), informação e uso de métodos contraceptivos, e motivos pessoais das gestantes relacionados a esse tema a fim de compreender e discutir como esses fatores podem contribuir para a ocorrência ou não da gestação na adolescência. Considera-se que investigar esses aspectos é importante para a construção de políticas públicas efetivas para a promoção da saúde do adolescente.

\section{Método}

\section{Participantes}

Participaram deste estudo 100 adolescentes entre 13 e 19 anos (50 grávidas primíparas $\mathrm{M}=17$; $\mathrm{DP}=1,77$ ) e (50 não grávidas $\mathrm{M}=16 ; \mathrm{DP}=1,75)$ que frequentavam instituições públicas da cidade de Santa Maria, interior do RS. Todas as adolescentes gestantes foram recrutadas em Unidades Básicas de Saúde do município. Já as adolescentes não grávidas, foram selecionadas em escolas públicas da mesma comunidade das gestantes. No momento da coleta de dados todas as adolescentes não-gestantes e apenas 23 (47\%) das gestantes adolescentes estavam estudando. A maioria das adolescentes gestantes possuía o ensino médio incompleto 22 (45\%). Do grupo das adolescentes não gestantes a maioria possuía o ensino fundamental incompleto 27 (55\%).

\section{Procedimentos de coleta de dados}

Os dados foram coletados, individualmente, nas UBS (coleta das gestantes) e, coletivamente, nas Escolas (não gestantes). Foi utilizado, como instrumento de coleta de dados um questionário, especialmente construído para este estudo, a partir da literatura sobre sexualidade na adolescência. Esse instrumento continha questões a respeito de dados sociodemográficos, informações sobre sexualidade, contracepção, gravidez e maternidade na adolescência e informações sobre métodos contraceptivos. Neste trabalho, foram utilizados os dados relativos à sexarca, uso e informações sobre métodos contraceptivos e afirmativas sobre motivações pessoais sobre o não uso de contracepção. Em relação à sexarca, foi realizada uma pergunta aberta sobre a idade em que aconteceu a primeira relação sexual, em ambos os grupos de adolescentes. A respeito do uso de contraceptivos, perguntou-se se houve uso de algum método na primeira relação sexual, tendo como opção (a) sim; (b) não e, (c) não lembro. Se a reposta fosse sim, a adolescente deveria responder qual método foi utilizado. Após, questionou-se acerca do uso de métodos contraceptivos nas relações sexuais posteriores: (a) sim, sempre, (b) não, nunca, (c) algumas vezes usei, outras vezes não usei. Se a adolescente respondeu que usou sempre ou algumas vezes, poderiam escolher uma ou mais entre as opções de contraceptivos: (a) camisinha masculina, (b) camisinha feminina, (c) contraceptivo oral, (d) coito interrompido, (d) DIU, (e) diafragma, (f) tabelinha e, (g) Outro, qual?

No que diz respeito às informações sobre métodos contraceptivos, as adolescentes foram questionadas se receberam, de cada pessoa - mãe, pai, irmão(ã), tio(a), amigo(a), professor(a), namorado, internet, TV, revistas: (a) nenhuma informação, (b) pouca informação, insuficiente para saber usar ou (c) informação suficiente para saber usar; pude tirar dúvidas. Após a resposta, as adolescentes foram perguntadas: Você considera as informações que recebeu sobre métodos contraceptivos suficientes para evitar uma gravidez? Sim ou não. Você gostaria de ter mais informação sobre métodos contraceptivos? Sim ou não. Que dúvidas você ainda tem? Nessa última pergunta as adolescentes poderiam falar sobre suas dúvidas. Já o instrumento que avaliou crenças sobre não uso de contracepção constituiu-se em afirmativas sobre motivações pessoais que justificavam o não uso de contraceptivos, como por exemplo. "Porque eu achava difícil que a gravidez fosse acontecer comigo"; Tinha medo que meus pais pudessem descobrissem"; "porque não gostava que eu usasse e ele não gostava de usar"; "eu achava que a pílula engordava". As adolescentes deviam marcar, se concordava ou não, com cada afirmativa.

Os dados foram analisados a partir do programa estatístico Statistical Package for the Social Sciences (SPSS) versão 13.0. Foram realizadas análises estatísticas dos resultados, conforme objetivos do estudo.

\section{Procedimentos éticos}

O projeto obteve aprovação da Secretaria Municipal de Saúde de Santa Maria e do Comitê de Ética em Pesquisa com Seres Humanos da Universidade Federal de Santa Maria, sob registro CAAE 0240.0.243.000-10. Antes da coleta de informações, obteve-se, por meio do Termo de Consentimento Livre e Esclarecido (TCLE), a autorização dos responsáveis por cada adolescente 
(gestante e não gestante) menor de 18 anos. Além disso, todas elas concordaram em participar do estudo, assinando o Termo de Assentimento ou TCLE. Assim, as Diretrizes e Normas que regulamentam as pesquisas envolvendo seres humanos (Parecer no 105/ 2006, com a Resolução 196/96, do Conselho Regional de Saúde/ MS, de 10/10/1996) foram respeitadas, durante a realização desta pesquisa

\section{Resultados e discussões}

A média de idade da sexarca no grupo gestantes foi $14,66(D P=1,52)$ e no grupo de não gestantes entre as jovens que já haviam tido a primeira relação sexual foi de 14,54 (DP=1,34). A fim de verificar diferenças de média na idade da sexarca entre os grupos foi realizado teste $\mathrm{t}$ de Student para amostras independentes. Os resultados da análise não indicaram diferenças estatisticamente significativas entre os grupos quanto à idade da sexarca $(t=0,373 ; g l=61 ; p=0,71)$. A idade da sexarca encontrada é similar a descrita em outros estudos realizados com adolescentes de camadas populares, que identificam a ocorrência do fenômento entre os 13 e 15 anos (Belo \& Silva, 2004; Marçal, Nunes, \& Dela Coleta, 2004; Trajman e cols., 2003; Vieira, Saes, Dória, \& Golberg, 2006).

Foi encontrado que $87 \%$ das gestantes e $100 \%$ das adolescentes não gestantes utilizaram métodos contraceptivos na primeira relação sexual. Não foram encontradas diferenças estatisticamente significativas entre esses grupos $(p=0,51)$, utilizando o teste qui-quadrado. Contudo, ao se comparar o uso de contraceptivos nas relações sexuais subsequentes, através do teste qui-quadrado, encontramos diferenças entres os grupos. A Tabela 1 exibe a frequência de respostas relacionadas ao uso de contraceptivos das adolescentes de ambos os grupos.

As adolescentes grávidas apresentaram um maior percentual de respostas associadas ao não uso ou uso infrequente de métodos contraceptivos nas relações sexuais subsequentes à sexarca $(58 \%$ indicaram usar algumas vezes e outras não e $8 \%$ nunca usaram) que as adolescentes não gestantes. Cabe lembrar que o uso infrequente de contraceptivos encontra-se tanto associado a maiores riscos de contração de DSTs como à ocorrência de uma gravidez não planejada (Alves \& Brandão, 2009; Brandão, 2009; Júnior e cols., 2009; Silva e cols., 2011). Não foi possível identificar diferenças em relação à presença de DSTs nos grupos, pois essa variável não foi objeto de investigação.

Os métodos contraceptivos utilizados na sexarca tanto por adolescentes gestantes como não gestantes foram: a camisinha masculina ( $72 \%$ gestantes e $89 \%$ não gestantes) e a pílula anticoncepcional oral $(63 \%$ gestantes e 36\% não gestantes). Também não foram identificadas diferenças estatisticamente significativas entre os grupos utilizando teste $t$ de student. Da mesma forma, o conhecimento e a utilização de outros métodos contraceptivos (coito interrompido, pílula do dia seguinte) foram similares nos grupos.

O mesmo foi encontrado nas relações sexuais subsequentes à sexarca, contudo observa-se que as adolescentes gestantes referem utilizar menos esses dois métodos contraceptivos. A camisinha masculina foi citada por $57 \%$ das gestantes e $89 \%$ das não gestantes, e o contraceptivo oral foi indicado por $39 \%$ das gestantes e 36\% das não gestantes, não sendo identificadas diferenças significativas entre os grupos $(p=0,75$ e $p=0,22$, respectivamente).

Ao serem questionadas sobre as informações de métodos contraceptivos, $96 \%$ das gestantes e $98 \%$ das não gestantes responderam que já receberam algum tipo de informação sobre o assunto, não havendo diferença estatisticamente significativa entre os grupos $(p=0,56)$. Entretanto, questiona-se a qualidade da informação recebida por essas jovens, uma vez que no grupo das gestantes o uso de métodos contraceptivos é infrequente.

De fato, alguns estudos (Alves \& Brandão, 2009; Beretta e cols., 2011; Brandão, 2009; Dias \& Gomes, 2000; Moura, Gomes, Rodrigues \& De Oliveira, 2011; Silva e cols., 2011; Spindola, Siqueira \& Cavalcanti, 2012; Vieira e cols., 2006) indicam que muitas adolescentes afirmam receber alguma informação sobre contracepção, no entanto, a informação é percebida por

Tabela 1. Frequência de uso de contraceptivo em outras relações sexuais

\begin{tabular}{lccc}
\hline & Gestantes (\%) & Não Gestantes (\%) & $p$ \\
\hline Usou sempre & 34 & 82 & $<0,01$ \\
Algumas vezes usou, outras não & 58 & 14 & $<0,01$ \\
Nunca usou & 8 & 4 & $<0,01$ \\
\hline
\end{tabular}

Psico-USF, Bragança Paulista, v. 19, n. 1, p. 13-22, jan./abril 2014 
elas como incompletas, parciais ou associadas a tabus e preconceitos, o que não contribui para sua utilização. Neste estudo, as adolescentes não foram questionadas sobre como as informações foram transmitidas.

Foi ainda investigada a quantidade de informação sobre contracepção recebida pelas diversas fontes possíveis de informação (mãe, pai, irmã, amiga, prima, tia, professora, namorado, revistas, TV, e internet), mediante uma escala de 3 pontos, a saber: nunca recebi informação alguma (valor 0), recebi informação insuficiente para saber usar (valor 1) e recebi informação suficiente para usar (valor 2). As adolescentes deveriam marcar uma das opções para cada informante. A Tabela 2 exibe as médias das respostas dos grupos das gestantes e das não gestantes. A comparação entre os grupos foi realizada por meio do teste $\mathrm{t}$ de Student. O tamanho do efeito ( $\mathrm{r}$ ) também é indicado na tabela.

As adolescentes gestantes descrevem que as mães são as que mais informam sobre métodos contraceptivos, seguidas pelas professoras e amigas. Já entre as não gestantes, a televisão, as amigas, a mãe, a internet, as revistas e a professora são as fontes mais citadas. As mães são mencionadas em ambos os grupos, porém apresentam um maior destaque enquanto fonte de informação entre as gestantes. Já o pai é poucas vezes citado nos dois grupos, tornando-se uma fonte quase inexistente de informação. Essas informações são similares a encontradas em outros estudos e podem revelar que a mãe parece estar presente na educação sexual das filhas e o pai ausente, em ambos os grupos (Fiorin,
Patias, \& Dias, 2011). Isso pode sobrecarregar as mães na tarefa de educar e informar as adolescentes.

O estudo de Alves e Brandão (2009), realizado com jovens de 18 a 24 anos de camadas populares que haviam tido pelo menos um episódio de gestação, revelou a importância atribuída ao papel da mãe como informante dos temas de sexualidade e contracepção. De acordo com os autores, as informações transmitidas pelas mães das adolescentes podiam servir de proteção ao exercício do comportamento sexual de risco. No presente estudo, percebe-se que a mãe foi citada nos dois grupos, sendo figura principal no grupo das gestantes. Apesar de terem sido citadas como principais informantes, essas informações não puderam servir como proteção ao comportamento sexual de risco, já que as gestantes estão grávidas.

Outra figura importante que foi citada nos dois grupos é a professora. A mesma recebeu destaque no grupo das gestantes. Pode-se pensar que à escola está cabendo a responsabilidade de fornecer, além da educação formal, educação informal sobre outros temas presentes em seu cotidiano (aprendizagem de regras para viver em sociedade) (Dessen \& Polônia, 2007; Gaspar, 2002). De fato, um estudo realizado por Polônia e Dessen (2005) revela que a família e a escola são dois contextos do desenvolvimento humano importantes, que apresentam tarefas específicas, devendo colaborar mutuamente. No entanto, atualmente pode estar ocorrendo um descompasso entre os sistemas, cabendo apenas à escola a realização de tarefas que

Tabela 2. Comparação entre os grupos sobre os informantes acerca dos métodos contraceptivos

\begin{tabular}{lllll}
\hline \multirow{2}{*}{ Fonte } & \multicolumn{2}{c}{ Média } & \\
\cline { 2 - 3 } Mẽestantes & Não gestantes & \multicolumn{1}{c}{ R } & 0,10 \\
Amigas & 1,37 & 1,17 & 0,28 & 0,03 \\
Professora & 1,18 & 1,23 & 0,78 & 0,11 \\
Namorado & 1,18 & 1,00 & 0,25 & 0,06 \\
Televisão & 0,96 & 0,84 & 0,52 & 0,20 \\
Tias & 0,94 & 1,25 & 0,71 & 0,06 \\
Revistas & 0,82 & 0,72 & 0,57 & 0,22 \\
Primas & 0,69 & 1,07 & 0,27 & 0,06 \\
Internet & 0,61 & 0,71 & 0,51 & 0,31 \\
Pai & 0,52 & 1,09 & $0,02 *$ & 0,05 \\
\hline
\end{tabular}

* $p<0,05$ - Nota: as médias podem variar de 0 a 2, conforme a seguinte escala de respostas utilizada: 0- Nenhuma informação; 1 - Pouca informação, insuficiente para saber usar; 2- Informação suficiente para saber usar; pude tirar dúvidas. 
seriam, primeiramente, da família (como a educação moral dos filhos - normas sociais). Nesse sentido, quando um dos contextos deixa a desejar, o outro pode se sobrecarregar e não cumprir adequadamente as tarefas (Carvalho, 2000; Dessen \& Polônia, 2007; Polônia \& Dessen, 2005).

As amigas também foram outra fonte de informação sobre métodos contraceptivos bastante citada entre os grupos. Pode-se considerar que, na adolescência, há uma maior aproximação e valorização do grupo de iguais, assim, é nesse ambiente que existem trocas de informações e experiências sobre sexualidade e contracepção (Knobel, 1981; Steinberg, 2010). Moura e cols., (2011) sugerem que a troca de informações sobre o tema entre amigas pode ser um fator de vulnerabilidade para as adolescentes. As informações repassadas pelas amigas podem começar de forma interessante, no entanto, podem partir para vulgarizações, comprometendo o conteúdo, fidedignidade e validade das mesmas (Moura e cols., 2011). Dias, Matos e Gonçalves (2007) também observam que os pares influenciam de maneira significativa no comportamento sexual dos jovens, podendo essa influência ser negativa, como, por exemplo, na situação de pressão para ter a primeira relação sexual. Alguns autores (Dias e cols., 2007; Steinberg, 2010) lembram que os adolescentes são cognitivamente imaturos para antecipar as consequências de seus atos, além de serem impulsivos, o que pode aumentar o risco de relação sexual desprotegida ou uso errado dos métodos contraceptivos, quando os amigos são os informantes.

O único meio de informação das adolescentes que possuiu diferença estatística significativa entre os grupos foi a internet $(p=0,02)$, com tamanho de efeito médio $(R=0,31)$. As adolescentes não gestantes são as que mais utilizam essa ferramenta como fonte de informação. A internet, até o presente momento, parece não ter alcançado a população gestante participante desta pesquisa. Pode-se levantar a hipótese de que as adolescentes do grupo de não gestantes possuem maior informação na internet, em função do maior acesso à mesma, estimulado pelas pesquisas propostas pela escola, que levam a jovem a usar esse recurso. Já as adolescentes gestantes que não estudam podem não se envolver no acesso a internet de maneira tão frequente.

Quando questionadas a respeito da qualidade da informação recebida, teste qui-quadrado indicou que $79 \%$ das adolescentes gestantes afirmaram que as informações recebidas foram suficientes para evitar uma gestação. Já entre as não gestantes, $80 \%$ consideraram que as informações recebidas eram suficientes para evitar uma gestação, não havendo diferenças estatisticamente significativas entre os grupos $(p=0,87)$. Os conhecimentos ou informações sobre o assunto não são os únicos fatores envolvidos na implementação de comportamentos contraceptivos adequados (Dias \& Gomes, 2000; Moura e cols., 2011; Dias \& Teixeira, 2010; Guimarães \& Witter, 2007).

Byrne (1983) revela que o uso de contraceptivos envolve cinco etapas: (a) retenção da informação; (b) reconhecimento da probabilidade do engajamento em alguma relação sexual; (c) seleção, obtenção e capacidade de uso correto do contraceptivo escolhido; (d) comunicação da decisão e escolha para o(a) parceiro(a), e (e) uso efetivo e competente do método selecionado. O autor salienta que os obstáculos ao uso dos métodos podem ocorrer em qualquer uma das etapas. Além disso, nem sempre a gestação é vista como algo indesejado ou inesperado na vida das adolescentes. Nesse estudo, por exemplo, 9 adolescentes grávidas (18\%) revelaram que não usavam métodos contraceptivos por uma motivação pessoal de desejo (consciente) de gestar.

Foi também questionado às adolescentes de ambos os grupos se elas gostariam de receber mais informações sobre métodos contraceptivos: $61 \%$ das gestantes e $72 \%$ das não gestantes gostariam de receber mais informações, não havendo diferença estatisticamente significativa entre os grupos $(\phi=0,31)$. Esses números são importantes e indicam que, apesar do aumento das informações, seja através da mídia, seja por intermédio dos familiares e amigos, ainda as adolescentes revelam necessidade de saber mais sobre o assunto (Spindola e cols., 2012; Vieira e cols., 2006). De fato, muitas informações são repassadas de maneira superficial, parcial ou incompleta, nem sempre havendo possibilidade de diálogo e reflexão sobre o tema (Alves \& Brandão, 2009; Dias \& Gomes, 2000).

Essa demanda de maiores informações talvez esteja tanto relacionada à forma quanto ao conteúdo das informações que são repassadas. Neste estudo, não investigamos como ocorreu a transmissão das informações, mas estudos (Leite e cols., 2004; Maheirie, Urnau, Vavassori, Orlandi \& Baierle, 2005; Soares, Amaral, Silva \& Silva, 2008) têm revelado que as informações repassadas pelos pais e pela escola têm sido imparciais e voltadas apenas para o uso de contraceptivos (quais são e como se usa). Não é permitido aos jovens um espaço de discussão sobre temas relacionados a sexualidade, embora parece ser esta uma demanda das jovens (Maheirie e cols., 2005; Soares e cols., 2008). 
Tabela 3. Motivos pessoais de adolescentes gestantes associados ao não uso de método contraceptivo

\begin{tabular}{|c|c|c|}
\hline Motivos & $\mathrm{n}$ & $\%$ \\
\hline Porque eu achava difícil que a gravidez fosse acontecer comigo. & 15 & 30 \\
\hline Tinha medo que meus pais pudessem descobrir. & 14 & 28 \\
\hline Porque já namoramos há algum tempo e eu pensei que não precisava. & 14 & 28 \\
\hline Nem pensava em usar contraceptivos. & 12 & 24 \\
\hline Porque transava pouco. & 10 & 20 \\
\hline Porque não dava tempo de usar algum método contraceptivo, quando víamos acontecia. & 10 & 20 \\
\hline Porque usar algum contraceptivo diminui o prazer. & 9 & 18 \\
\hline Porque achava que a pílula engordava. & 9 & 18 \\
\hline Porque nós não tínhamos camisinha. & 9 & 18 \\
\hline Porque eu achava que não precisa usar contraceptivos, pois eu era muito nova para engravidar. & 9 & 18 \\
\hline Eu não usava porque queria engravidar. & 9 & 18 \\
\hline Porque comecei a usar anticoncepcional, mas me fez mal. & 7 & 14 \\
\hline Porque se eu usasse algum método contraceptivo os outros iam pensar que eu transava com qualquer um. & 6 & 12 \\
\hline Porque já namoramos há algum tempo e eu achava que usar era sinal de desconfiança. & 6 & 12 \\
\hline Não tinha dinheiro para comprar. & 5 & 10 \\
\hline Porque o parceiro não gostava que eu usasse e ele não gostava de usar. & 5 & 10 \\
\hline
\end{tabular}

Nota: Nessa questão foi permitido marcar mais de uma opção de resposta; por isso as frequências não são cumulativas, devendo ser interpretadas separadamente para cada motivo.

Por outro lado, estudos têm indicado que não apenas informações adequadas e corretas são capazes de efetivar o comportamento contraceptivo dos adolescentes, mas que motivações pessoais implicam maior ou menor utilização de métodos contraceptivos (Brandão, 2009; Heilborn e cols., 2002; Taquette e cols., 2004). De fato, estudos apontam que a não adoção de medidas contraceptivas entre adolescentes é fortemente influenciada pelas questões de gênero, por exemplo. A negociação no uso do contraceptivo acaba por colocar a mulher, muitas vezes, no papel de submissão. Por outro lado, se a mulher prepara-se para o intercurso sexual, de forma a proteger-se, pode ver-se mal diante dos olhos masculinos (Brandão, 2009). Além disso, o tempo de relacionamento, se parceiro casual ou fixo, também influenciam nas práticas contraceptivas (Almeida \& Hardy, 2007; Heilborn e cols., 2002). Outros aspectos que também podem diminuir o uso são a dificuldade de acesso aos métodos, como a falta de dinheiro para comprar, ou medo e vergonha de ir aos serviços públicos que distribuem os contraceptivos, além de crenças sobre seus efeitos colaterais (Brandao, 2009). Esses aspectos foram investigados em nosso estudo e talvez possam justificar o motivo pelo qual as adolescentes gestantes, mesmo possuindo informações sobre métodos contraceptivos, não fizeram o uso delas. A partir de um instrumento com afirmativas sobre os possíveis motivos associados ao não uso de métodos contraceptivos, foi realizada uma análise de frequência sobre as respostas das gestantes.

Os resultados demonstraram uma diversidade de motivações pessoais decorrentes tanto de aspectos individuais e próprios da adolescência, como pensamento mágico e crença de invulnerabilidade de que "a gravidez não acontece comigo" até aspectos sociais e culturais relativos ao gênero feminino ("o que os outros vão pensar", se estiver usando algum método e não estiver namorando). Esses aspectos demonstram a multiplicidade de fatores implicados na decisão do uso ou não do contraceptivo que podem ter contribuído para que as gestantes tenham engravidado.

\section{Conclusões}

Neste estudo foram investigadas algumas variáveis que podem contribuir para a presença de uma maior vulnerabilidade para a ocorrência de uma gestação durante a adolescência, a saber: a idade da primeira 
relação sexual (sexarca), informação e uso de métodos contraceptivos nas relações sexuais, além de motivações pessoais das gestantes. Foram comparados dois grupos de adolescentes (gestantes e não gestantes) com o intuito de verificar se havia diferenças nas mesmas entre os dois grupos. Percebeu-se que não houve diferença significativa na idade da sexarca, nem em relação ao parceiro da primeira relação sexual. No que diz respeito ao uso de métodos contraceptivos na sexarca, também não houve diferença significativa entre as jovens que já haviam tido relação sexual nos dois grupos. No entanto, o uso de contraceptivos nas relações sexuais subsequentes à sexarca apresentou diferença estatisticamente significativa entre os grupos $(p<0,01)$, sendo o uso mais frequente de contraceptivo no grupo das não gestantes. No grupo das gestantes, verificou-se o uso infreqüente de métodos, além de motivações pessoais e crenças muitas vezes errôneas sobre métodos contraceptivos, que podem estar associados à maior vulnerabilidade dessas jovens à gestação.

Cabe ressaltar que, nos dois grupos de adolescentes, os contraceptivos mais citados foram a camisinha masculina e o contraceptivo oral (pílula anticoncepcional), o que confirma achados de outros estudos realizados com adolescentes (Alves \& Brandão, 2009; Beretta e cols., 2011; Moura cols., 2011; Vieira cols., 2006). Uma informação importante, que não foi questionada neste estudo e que fica de sugestão para a realização de próximos estudos, é se o uso do contraceptivo oral ocorria concomitantemente à utilização da camisinha masculina ou não, pois esse segundo método é o que protege contra as DSTs.

Ambos os grupos revelaram receber informações sobre métodos contraceptivos. Por outro lado, as jovens revelam que queriam saber mais sobre os mesmos. Pode-se pensar que esse desejo advenha do fato de as informações que elas obtiveram poderiam ter sido repassadas de forma imparcial ou tratarem apenas de como usar os métodos contraceptivos, sem oferecer um espaço para questionamentos, reflexões e discussões sobre outras questões relacionadas à sexualidade. De fato, muitos estudos têm revelado que não há espaço de reflexão sobre corpo, sexualidade e afetividade (Dias \& Gomes, 2000; Júnior e cols., 2009). Talvez isso explique por que muitas adolescentes engravidaram, mesmo tendo informações sobre contracepção. Ainda, é preciso ir além da discussão da presença ou ausência de informações para pensar a ocorrência da gestação na adolescência (Dias \& Teixeira, 2010). Políticas públicas voltadas à população jovem devem considerar projetos de vidas, além de desejos e anseios dos jovens sobre sexualidade, como forma de tornar suas ações mais efetivas.

Em relação a políticas públicas para a população jovem, tem-se o Programa de Saúde do Adolescente (PROSAD), que possui o objetivo de promover a saúde integral do adolescente, favorecendo seu crescimento e desenvolvimento, por meio de diferentes práticas de tratamento, prevenção e promoção de saúde (Brasil, 1996). Dentre sua atuação, encontram-se ações voltadas para a saúde sexual dos jovens, mediante a implantação de programas estaduais e municipais. A atuação do PROSAD ultrapassaria as unidades básicas de saúde e hospitais, agindo em escolas, centros comunitários, clubes e outros locais que os jovens frequentam. Contudo, muitos profissionais desconhecem o programa. Além disso, em alguns casos, quando há ações referentes à sexualidade e contracepção, essas restringem-se a passagens de informações e distribuição de métodos contraceptivos. Não há a oferta de apoio e diálogo aos adolescentes, conforme previsto no programa (Santos \& Arpini, 2010).

Ao mesmo tempo em que se percebem impasses nas políticas públicas para o público jovem, em virtude de dificuldades dos profissionais que atuam junto a ele, os pais dos adolescentes também parecem encontrar dificuldades no desenvolvimento de uma educação sexual dos filhos. Nosso estudo indica que a mãe pode estar sendo sobrecarregada com essa atividade, já que é o único membro da família que parece oferecer uma orientação maior junto às jovens. Destaca-se que, no instrumento de pesquisa, as jovens podiam ainda indicar pai, irmãs e tias como fontes de informação.

Poucos são os programas voltados aos pais, para ensiná-los a como dialogar, tirar dúvidas e orientar os filhos no que se refere à sexualidade e contracepção. Estudos mostram que os pais apresentam dificuldades em lidar com o assunto, pois não tiveram a experiência de conversar com os próprios pais sobre sexualidade; assim lhes faltam modelos e repertórios de estratégias para abordar o tema com os filhos (Dias \& Gomes, 2000). Além disso, o próprio tema da sexualidade adolescente, em nossa sociedade, é tratado comumente como algo que deve ser censurado e/ou controlado (Louro, 2000).

Os profissionais da escola também parecem não se encontrar preparados para colaborar com os pais na tarefa da educação sexual dos jovens (Maheirie e cols., 2005; Soares e cols., 2008) No entanto, em nosso estudo, os professores foram citados entre os principais 
informantes, o que nos sugere que a escola também deve fazer parte das políticas públicas. Para tanto, os professores, supervisores e orientadores educacionais devem estar preparados para ajudar os jovens.

A universidade pode ter um papel importante nesse sentido, promovendo, por meio de atividades voltadas à comunidade, como programas de treinamento de pais e professores, treinamento de habilidades sociais (assertividade) voltados aos jovens, oficinas e grupos operativos que visem sensibilizar ou discutir sobre temas como corpo, afetividade, sexualidade e contracepção. Destaca-se que essas atividades não podem se restringir apenas ao público adolescente, uma vez que diferentes atores que participam do desenvolvimento dos jovens também apresentam dificuldades em dialogar ou trabalhar com esses temas.

Além disso, ressalta-se que para trabalhar com o público adolescente deve-se ampliar a forma como se trabalha o fenômeno da gravidez na adolescência, bem como a sexualidade. Trabalhar de forma ampliada quer dizer que a sexualidade e o fenômeno da gravidez devem ser considerados para além da informação, de forma a imprimir o foco na prevenção da gravidez e promoção de saúde, quando ela já aconteceu. É importante que os profissionais que trabalhem com o público adolescente tenham ciência de que a sexualidade faz parte do ser humano e que os jovens devem ser instruídos de forma a vivenciar sua sexualidade de maneira mais saudável. Para isso, é necessário ouvir o que os jovens têm a falar sobre o assunto, principalmente no que diz respeito as suas motivações para não uso ou uso dos contraceptivos. A partir disso, podem-se construir políticas públicas e intervenções mais eficazes tanto na prevenção como na promoção da saúde adolescente.

\section{Referências}

Almeida, A., \& Hardy, E. (2007). Vulnerabilidade de gênero para a paternidade em homens adolescentes. Revista de Saúde Pública, 41(4), 565-572.

Alves, C. A., \& Brandão, E. R (2009). Vulnerabilidades no uso de métodos contraceptivos entre adolescentes e jovens: interseções entre políticas públicas e atenção à saúde. Ciência \& Saúde Coletiva, 14, 661-670.

Asinelli-Luz, A., \& Junior, F. N. (2008). Gênero, adolescência e prevenção ao HIV/AIDS. Pro-posições, 19, 81-97.
Belo, M. A., \& Silva, J. L. (2004). Conhecimento, atitude e prática sobre métodos anticoncepcionais entre adolescentes gestantes. Revista de Saúde Pública, 38, 479-487.

Beretta, M. I., Clápis, C. V., Neto, L. A., Freitas, M. A., Dupas, G., Ruggiero, E. M., \& Baltor, M. R. (2011). A contextualização da gravidez na adolescência em uma maternidade de São Carlos/SP. Revista Eletrônica de Enfermagem, 13, 90-98.

Brandão, E. (2009). Desafios da contracepção juvenil: interseções entre gênero, sexualidade e saúde. Ciência \& Saúde Coletiva, 14, 1063-1071.

Brasil (1996). Programa saúde do adolescente. Bases programáticas. $2^{a}$ ed. Secretaria Executiva. Coordenação da Saúde da Criança e do Adolescente. Brasília, Ministério da Saúde.

Byrne, D. (1993). Personality and attitudinal barries to contraception. Em D. Byrne, \& Fisher, W. A. (Eds). Adolescents, sex and contraception. (pp. 3-31). NJ: Erlbaum.

Cano, M. A. T., Ferriani, M. G. C., \& Gomes, R. (2000). Sexualidade na adolescência: um estudo bibliográfico. Revista Latino-Americana de Enfermagem, 8, $18-24$.

Carvalho, M. E. (2000). Relações entre familia e escola e suas implicações de gênero. Cadernos de Pesquisa, 110, 143-155.

Carvalho, M. L. O., \& Schor, N. (2005). Motivos de rejeição aos métodos contraceptivos reversíveis em mulheres esterilizadas. Revista de Saúde Pública, 39(5), 788-794.

Charles, V. E., \& Blum, R. W. (2008). Core competencies and the prevention of high-risk sexual behavior. Em N. G. Guerra, \& C. P. Bradshaw (Eds.), Core competencies to prevent problem behaviors and promote positive youth development. New directions for child and adolescent development, 122, 61-74.

Cruzeiro, A. L., Souza, L. D., Da Silva, R. A., Pinheiro, R. T., Da Rocha, C. L., \& Horta, B. L. (2010). Comportamento sexual de risco: fatores associados ao número de parceiros sexuais e ao uso de preservativo em adolescentes. Ciência \& Saúde Coletiva, 15,1149-1158.

Dessen, M. A., \& Polônia, A. C. (2007). A família e a escola como contextos de desenvolvimento humano. Paidéia, 17, 21-32. 
Dias, A. C. G., \& Gomes, W. B. (2000). Conversas, em familia, sobre sexualidade e gravidez na adolescência: a percepção de jovens gestantes. Psicologia: Reflexão e Crítica, 13, 109-125.

Dias, A. C. G., \& Teixeira, M. A. P. (2010). Gravidez na adolescência: um olhar sobre um fenômeno complexo. Paidéia, 45,123-131.

Dias, S., Matos, M., \& Gonçalvez, A. (2007). Percepção dos adolescentes acerca da influência dos pais e pares nos seus comportamentos sexuais. Análise Psicológica, 4, 625-634.

Fiorin, P., Patias, N. D., \& Dias, A. C. G. (2011). Reflexões sobre a mulher contemporânea e a educação dos filhos. Sociais e Humanas, 24(2), 121-132.

Gaspar, A. (2002). A educação formal e a educação informal em ciências. Em L. I. Massarani, I. Moreira, \& F. Brito. (Orgs). Ciência e público: caminhos da divulgação científica no Brasil. (pp.71-183). Rio de Janeiro: Casa da Ciência.

Guimarães, E. A., \& Witter, G. P. (2007). Gravidez na adolescência: conhecimentos e prevenção entre jovens. Boletim Academia Paulista de Psicologia, 27, 167-180.

Heilborn, M. L., Salem, T., Rohden, F., Brandão, E., Knauth, D., Víctora, C., Aquino, E., McCallum, C., V. \& Bozon, M. (2002). Aproximações socioantropológicas sobre a gravidez na adolescência. Horizontes Antropológicos, 8(17), 13-45.

Júnior, J. C., Nahas, M. V., Barros, M. V., Loch, M. R., Oliveira, E. S., De Bem, M. F., \& Lopes, A. S. (2009). Comportamentos de risco à saúde em adolescentes no Sul do Brasil: prevalência e fatores associados. Revista Panamericana de Salud Publica, 25, 344-352.

Knobel, M. (1981). Síndrome da adolescência normal. Em A., Aberastury, \& M. Knobel. Adolescência normal: um enfoque psicanalítico. (pp. 24-62). Porto Alegre: Artes Médicas.

Kotchick, B., Shaffer, A., Forehand, R., \& Miller, K. (2001). Adolescent sexual risk-behavior: a multi-system perspective. Clinical Psychology Review, 21, 493-519.

Leite, I. C., Rodrigues, R. N., \& Fonseca, M. C. (2004). Fatores associados com o comportamento sexual e reprodutivo entre adolescentes das regiões Sudeste e Nordeste do Brasil, Cadernos de Saúde Pública, 20, 474-481.
Louro, G. L. (2000). Pedagogias da sexualidade. Em G.L., Louro, J., Weeks, D., Britzman, R. Parker, \& J. Butler. O corpo educado: pedagogias da sexualidade. (pp. 3-22). Autêntica, Belo Horizonte.

Luster, T., \& Small, S. (1994). Factors associated with sexual risk-taking behaviors among adolescents. Journal of Marriage and the Family, 56, 622-632.

Maheirie, K., Urnau, L. C., Vavassori, M. B., Orlandi, R., \& Baierle, R. E. (2005). Oficinas sobre sexualidade com adolescentes: um relato de experiência. Psicologia em Estudo, 10, 537-542.

Marçal, V. P., Nunes, L. G., \& Dela Coleta, M. F. (2004). Estudo sobre crenças e comportamento sexual de adolescentes. Revista Eletrônica da Sociedade de Psicologia do Triângulo Mineiro, 92, 143-149.

Moura, L. N., Gomes, K. R., Rodrigues, M. T., \& De Oliveira, D. C. (2011). Informações sobre contracepção e sexualidade entre adolescentes que vivenciaram uma gravidez. Acta Paulista de Enfermagem, 24, 320-326.

Oliveira, S. H., Dias, M. R., \& Silva, M. I. (2005). Adolescentes e aids: fatores que influenciam a intenção de uso do preservativo. Jornal Brasileiro de Doenças Sexualmente Transmissiveis, 17, 32-38.

Polônia, A. C., \& Dessen, M. A. (2005). Em busca de uma compreensão das relações entre família-escola. Psicologia Escolar e Educacional, 9, 303-312.

Santos, A., \& Carvalho, C. V. (2006) Gravidez na adolescência: um estudo exploratório. Boletim de Psicologia, LVI, 135-151.

Santos, B. R., \& Arpini, D. M. (2010). Estratégia da saúde da família e o atendimento aos adolescentes. Dissertação de mestrado em psicologia (não publicada). Universidade Federal de Santa Maria (UFSM), Santa Maria, RS.

Silva, K. S., Rozenberg, R., Bonan. C, Chuva, V. C., Da Costa, S. F., \& Gomes, M. A. (2011). Gravidez recorrente na adolescência e vulnerabilidade social no Rio de Janeiro (RJ, Brasil): uma análise dos dados do Sistema de Nascidos Vivos. Ciência \& Saúde Coletiva, 16, 2485-2493.

Soares, S. M., Amaral, M. A., Silva, L. B., \& Silva, P. A. B. (2008). Oficinas sobre sexualidade na adolescência: revelando vozes, desvelando olhares de estudantes de ensino médio. Escola Anna Nery Revista de Enfermagem, 12, 485-491. 
Spindola, T., Siqueira, N. S., \& Cavalcanti, R. L. (2012). As gestantes adolescentes e o emprego dos métodos contraceptivos. Revista de pesquisa: Cuidado é Fundamental Online, 4, 2636-2646.

Steinberg, L. (2010). A dual system model of adolescent risk-taking. Developmental Psychobiology, 52, 216-224.

Taquette, S., Andrade, R., Vilhena, M., \& De Paula, M. (2005). A relação entre as características sociais e comportamentos da adolescente e as doenças sexualmente transmissíveis. Revista Associação Médica Brasileira, 51, 148-152.

Taquette, S., \& Vilhena, M. (2008). Uma contribuição ao entendimento da iniciação sexual feminina na adolescência. Psicologia em Estudo, 13,105-114.

Taquette, S., Vilhena, M., \& Paula, M. C. (2004). Doenças sexualmente transmissíveis e gênero: Um estudo transversal com adolescentes do Rio de Janeiro. Cadernos de Saúde Pública, 20(1), 282-290.

Trajman, A., Belo, M. T., Teixeira, E. G., Dantas, V. C., Salomão, F. M., \& Cunha, A. J. (2003). Knowledge about SDT/AIDS and sexual behavior among hight school students in Rio de Janeiro, Brazil. Cadernos de Saúde Pública, 19,127-133.

Vieira, L. M., Saes, S. O., Dória, A. A, \& Golberg, T. B. (2006). Reflexões sobre a anticoncepção na adolescência no Brasil. Revista Brasileira de Saúde Materno Infantil, 6, 135-140.

Villela, W. V., \& Doretto, D. T. (2006). Sobre a experiência sexual dos jovens. Cadernos de Saúde Pública, 22, 2467-2472.

Ximenes Neto, F. R., Dias, M. S., Rocha, J., \& Cunha, I. C. (2007). Gravidez na adolescência: motivos e percepções das adolescentes. Revista Brasileira de Enfermagem, 60, 279-285.

Recebido em: 18/12/2012

Reformulado em: 02/07/2013

Segunda reformulação em: 12/11/2013

Aprovado em: 21/11/2013

Sobre as autoras:

Naiana Dapieve Patias é psicóloga, especialista em Criança e Adolescente em Situação de Risco (UNIFRA) e mestre em Psicologia (UFSM).

E-mail: naipatias@hotmail.com

Ana Cristina Garcia Dias é psicóloga, doutora em Psicologia (USP) e docente do curso de Pós-graduação em Psicologia (UFSM).

E-mail: anacristinagarciadias@gmail.com

Este trabalho faz parte da dissertação de mestrado da primeira autora, orientada pela co-autora no Programa de pós-graduação em Psicologia da Universidade Federal de Santa Maria (UFSM). As autoras agradecem o apoio financeiro concedido através de bolsa Capes à autora e auxílio financeiro CNPq através do edital MCT/CNPq/MEC/Capes n 2/2010 ao projeto "Práticas educativas parentais vividas por adolescentes grávidas e não grávidas". 